\title{
Magnetoencephalographic Source Localization of the Eye Area of the Motor Homunculus
}

\author{
Richard Wennberg, Luis Garcia Dominguez, J. Martin del Campo
}

\begin{abstract}
A patient with intractable epilepsy, previous right frontal resection, and active vagus nerve stimulation (VNS) developed new onset quasi-continuous twitching around the left eye. Electroencephalography showed no correlate to the orbicularis oculi twitches apart from myographic potentials at the left supraorbital and anterior frontal electrodes. Magnetoencephalography was performed using spatiotemporal signal space separation to suppress magnetic artifacts associated with the VNS apparatus. Magnetoencephalographic source imaging performed on the data back-averaged from the left supraorbital myographic potentials revealed an intrasulcal cortical generator situated in the posterior wall of the right precentral gyrus representing the eye area of the motor homunculus.
\end{abstract}

RÉSUMÉ: Localisation par magnétoencéphalographie de la zone oculaire qui correspond à l'homoncule moteur. Un patient atteint d'épilepsie réfractaire chez qui on avait pratiqué une résection frontale du côté droit du cerveau et qu'on avait soumis à une stimulation neuro-vagale (SNV) a fini par développer des contractions musculaires quasi-continues autour de l'œil gauche. Hormis des potentiels myographiques détectés par des électrodes situées dans les régions supraorbitale gauche et frontale antérieure, un électroencéphalogramme (EEG) n'a révélé aucun phénomène corrélatif en lien avec les contractions du muscle orbiculaire de l'œil. Un examen de magnétoencéphalographie (MEG) a été ensuite réalisé au moyen d'une rupture de l'espace entre les signaux spatio-temporels afin d'étouffer les artefacts magnétiques associés à l'appareil de SNV. L'origine de l'imagerie de l'examen de MEG réalisé à partir des données moyennes correspondant aux potentiels myographiques de la région gauche supraorbitale a révélé un générateur cortical situé dans la paroi postérieure du gyrus précentral, lequel représente en fin de compte la zone oculaire correspondant à l'homoncule moteur.

Keywords: Electroencephalography (EEG), Epilepsia partialis continua (EPC), MEG source imaging (MSI), Orbicularis oculi, Spatiotemporal signal space separation (tSSS), Vagus nerve stimulation (VNS)

doi:10.1017/cjn.2018.373

Can J Neurol Sci. 2019; 46: 108-114

A 35-year-old woman was referred to the epilepsy monitoring unit (EMU) because of intractable focal motor seizures and a 3month history of twitching of the left eye. Epilepsy was diagnosed at age 22 years; seizures commencing with left arm tingling and then jerking of the arm, followed by left leg tingling and jerking, with or without secondary generalization. Seizures proved to be medically intractable, and 5 years later she underwent a partial right frontal lobe resection after intracranial electroencephalography (EEG) monitoring, the latter documenting not only frontal lobe epileptiform activity but also independent temporoparietal abnormalities (details in Supplementary Material, accessible at journals.cambridge.org). Pre- and peri-natal and developmental history were normal, pre-surgical MRI brain scans were normal, and surgical pathology showed no definite abnormalities in the resected frontal lobe tissue.

Seizure relief was only transient post-resection, and 4 years later a vagus nerve stimulator (VNS) was implanted, without clear benefit in terms of seizure control. At the time of this EMU admission, the patient was receiving levetiracetam $4000 \mathrm{mg} /$ day, lacosamide $400 \mathrm{mg} /$ day, topiramate $400 \mathrm{mg} / \mathrm{day}$, and eslicarbazepine $400 \mathrm{mg} / \mathrm{day}$. Her VNS was programmed to cycle 30 seconds on $/ 3$ minutes off, $25 \mathrm{~Hz}, 1 \mathrm{~mA}, 500$ mseconds pulse width.

In the EMU, interictal EEG revealed sporadic multifocal epileptiform discharges, predominating over the right central region and, independently, over the right temporal region, the latter focus often showing evidence of complex posterior to anterior spike propagation. Remarkably, the most prominent feature of the EEG recordings was the presence of nearly continuous high-amplitude arrhythmic spikes over the left anterior frontal region, with positive phase reversal in bipolar montages at F3 and maximal amplitude in referential montages at Fp1 (electronegative) $>$ F3 (electropositive). Review of the video recording supported

From the Division of Neurology (RW, LGD, JMdC) and the Mitchell Goldhar MEG Unit (RW, LGD), Krembil Brain Institute, University Health Network, Toronto Western Hospital, University of Toronto, Toronto, Ontario, Canada.

Received SePtember 10, 2018. Final Revisions Submitted October 12, 2018. Date of ACCEPTANCE OCTOBER 19, 2018.

Correspondence to: R. Wennberg, Toronto Western Hospital, 399 Bathurst Street, Suite 5W444, Toronto, ON, Canada M5T 2S8. Email: richard.wennberg@uhn.ca 


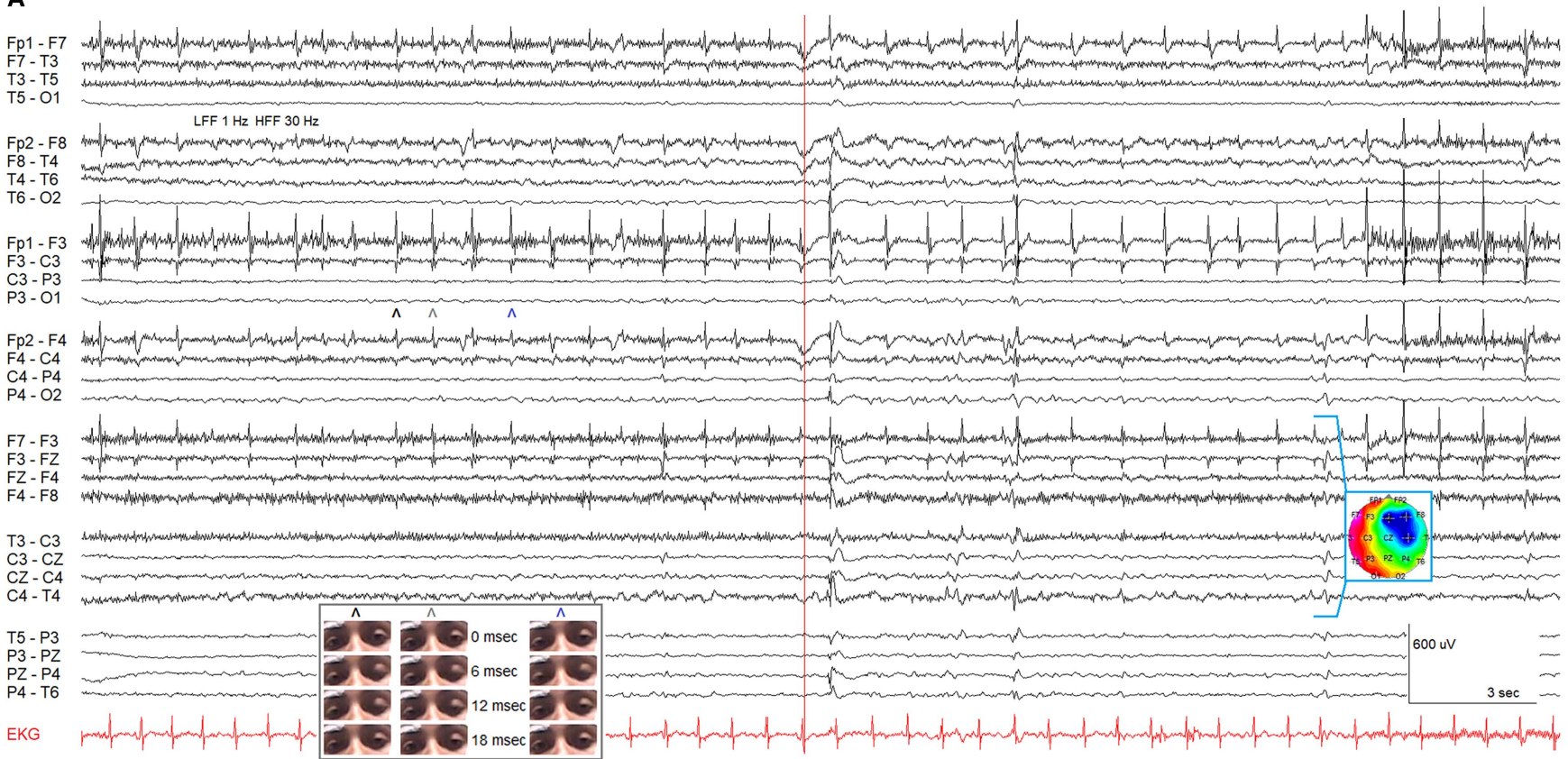

B

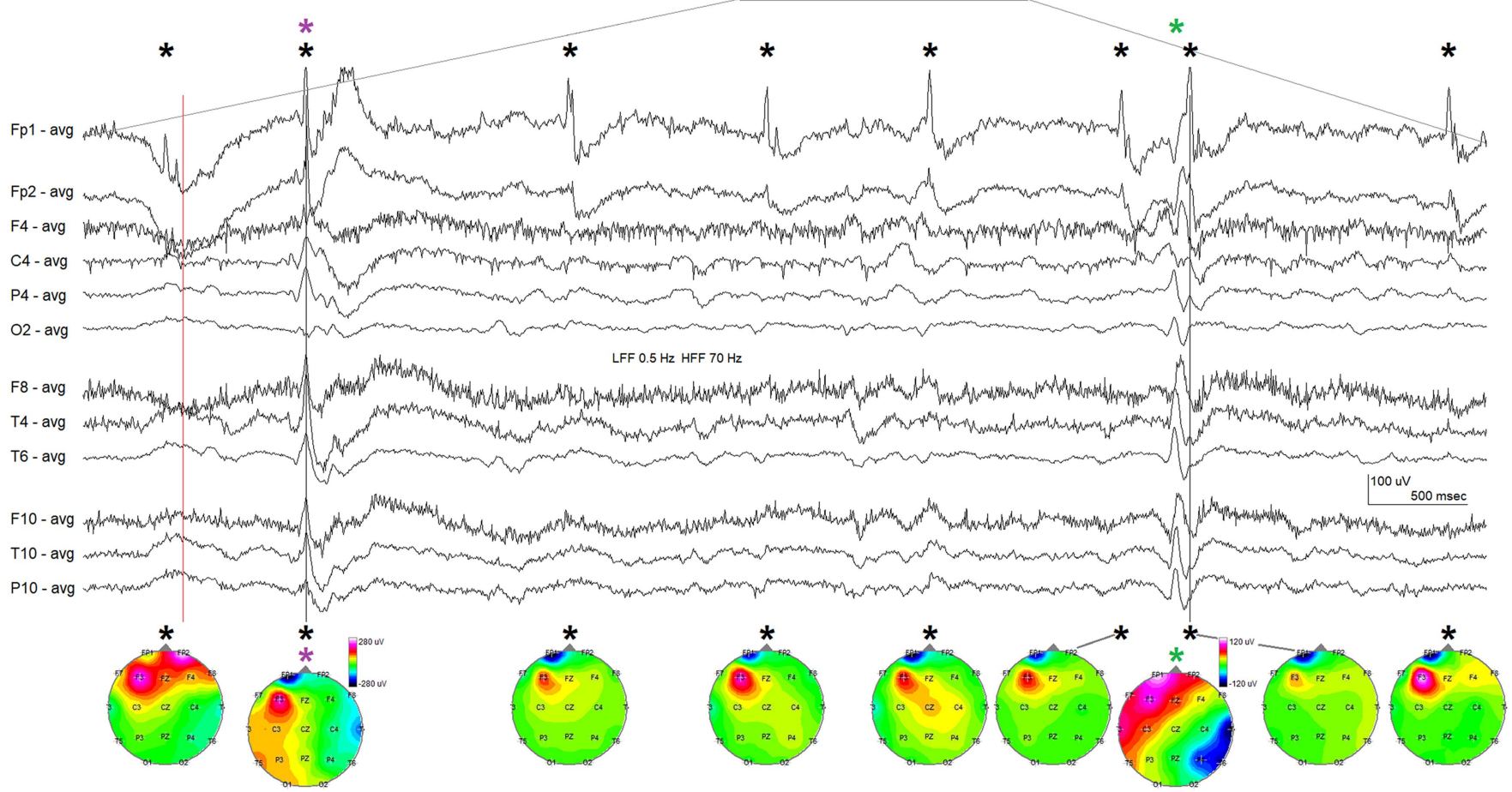

Figure 1: (A) EEG sample from EMU recording acquired with patient's eyes open (before red vertical line) and eyes closed (after red vertical line). The recording is dominated by extremely high-amplitude arrhythmic left frontal spike potentials (positive phase reversal at F3, maximal (negative) amplitude at F 1). Longitudinal and transverse bipolar montage. Left inset shows freeze frame video screen captures, at 3-msecond intervals (top to bottom), of the eye twitches seen in association with the three left frontal (myographic) spike discharges marked by^. The left eyelid can be seen in each case to close fully, whereas the right eyelid only partially closes. (The video [MP4 format] "Orbicularis oculi contractions and EEG" that accompanies this EEG sample is available as Supplementary Material, accessible at journals.cambridge.org). Right inset shows voltage topography of a right frontocentral interictal epileptiform discharge (underlined). (B) Selected channels from the underlined section of EEG in (A) depicted in common average reference montage at slower sweep speed. Just after eye closure (red vertical line), a left lower face myoclonic jerk occurred synchronously with an eye twitch and a right temporal maximal epileptiform discharge (purple asterisk, first gray vertical line). Some similar clinical occurrences, however, were not associated with corresponding epileptiform discharges (see, e.g. the first eye twitch of the EEG sample in (A) and the accompanying Supplementary Material video). A myoclonic jerk of the left hand was associated with a complex right temporal maximal epileptiform discharge (green asterisk), not synchronous with an orbicularis contraction shortly after (second gray vertical line). At bottom, EEG voltage topographic plots at the peak of the myographic potentials (black asterisks) are dominated by the left Fpl (blue, negative) and F3 (red, positive) electromyographic fields (and by frontal electropositive upward eye movement artifact associated with volitional eye closure [red vertical line] in the first instance). The first right temporal spike (purple asterisk, first gray vertical line) has minimal topographic representation compared to the synchronous left frontal electromyographic field. The second right temporal epileptiform discharge, however, accompanying the hand myoclonic jerk but unassociated with a synchronous eye twitch does show a right hemispheric, temporal maximal voltage topography (green asterisk). EEG = electroencephalography; EMU = epilepsy monitoring unit. 


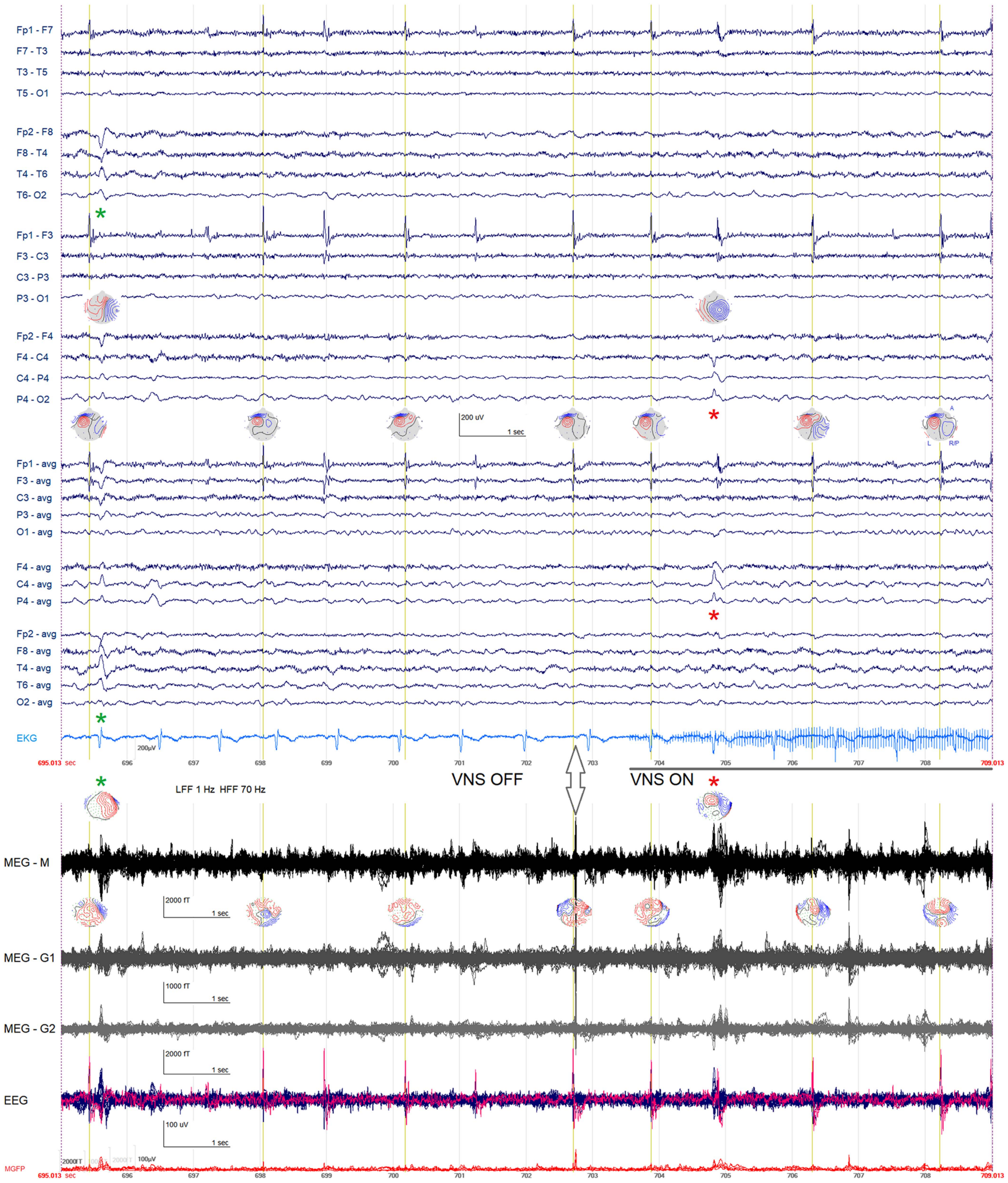

Figure 2: Segment of EEG/MEG recording showing frequent left frontal myographic discharges apparent in EEG at Fp1 (negative, blue topographic distribution) and F3 (positive, red topographic distribution) as well as a right temporal interictal epileptiform discharge (green asterisk) and a right central interictal epileptiform discharge (red asterisk). Eyes closed, drowsy state. The butterfly plots at bottom show the simultaneous MEG recording, including 102 magnetometer channels (M), 102 planar gradiometer channels (G1), and 102-orthogonal to G1planar gradiometer channels (G2) as well as the EEG channels including supraorbital (SO1/SO2) electrodes (pink). The vagus nerve stimulator $(V N S)$ is off during the initial portion of the recording segment but turns on during the later portion (evident as $25 \mathrm{~Hz}$ artifact in the EKG lead). There is a transient magnetic artifact apparent before the stimulation artifact (double headed arrow), presumably related to the device activating its soft start mechanism. The magnetometer flux fields recorded in association with individual, unaveraged eye twitches (red = magnetic flux out of the head, blue = magnetic flux into the head) do not show a consistent pattern before or after VNS activation, but the flux fields associated with the two interictal spikes are compatible with the temporal and central EEG voltage topographic plots. Yellow vertical lines indicate myographic potentials selected for back-averaging for MEG source localization. EEG=electroencephalography; MEG=magnetoencephalography; $M G F P=$ mean global field power. 
interpretation of the high-amplitude frontal spikes as myographic potentials associated with contractions of the left orbicularis oculi muscle (Figure 1A; corresponding video [MP4 format] available as Supplementary Material). Synchronous lower amplitude myographic potentials over the right frontopolar region at Fp2 were apparent when the patient's eyes were open and correlated with partial activation of the right orbicularis occurring synchronously with the left orbicularis contractions (Figure 1A, inset, and video). The right frontal myographic potentials were much less evident with the eyes closed.
Apart from the myographic electrical field present at Fp1-F3, no EEG correlate could be appreciated with the eye twitches (Figure 1B). Infrequently, an orbicularis contraction was associated with a synchronous myoclonic twitch of the left lower face, usually with no EEG correlate but on rare occasion accompanied by a synchronous spike maximal at T4 (Figure 1B, and video). Separately, occasional myoclonic jerks of the left hand occurred, with or without an EEG correlate in the form of a propagating temporal maximal spike (Figure 1B). The hand twitches were not synchronous with the eye twitches.
A

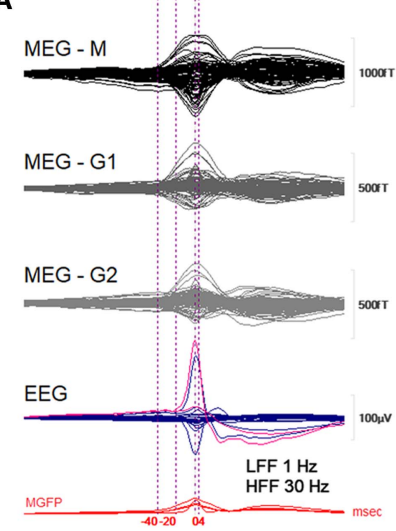

B
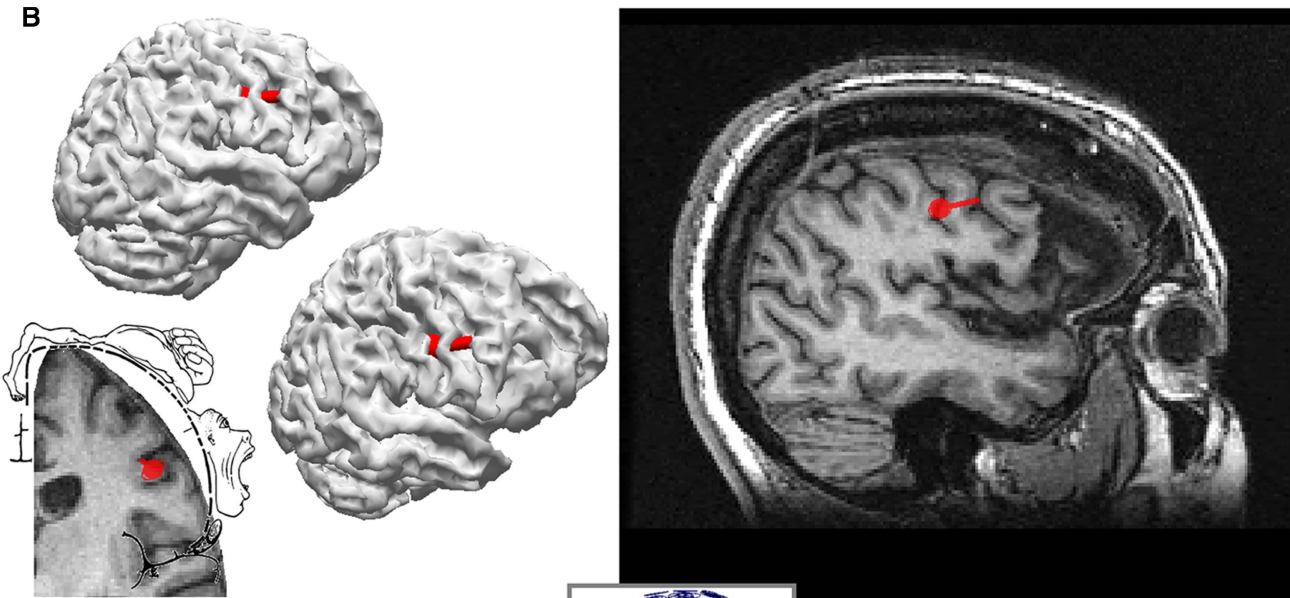
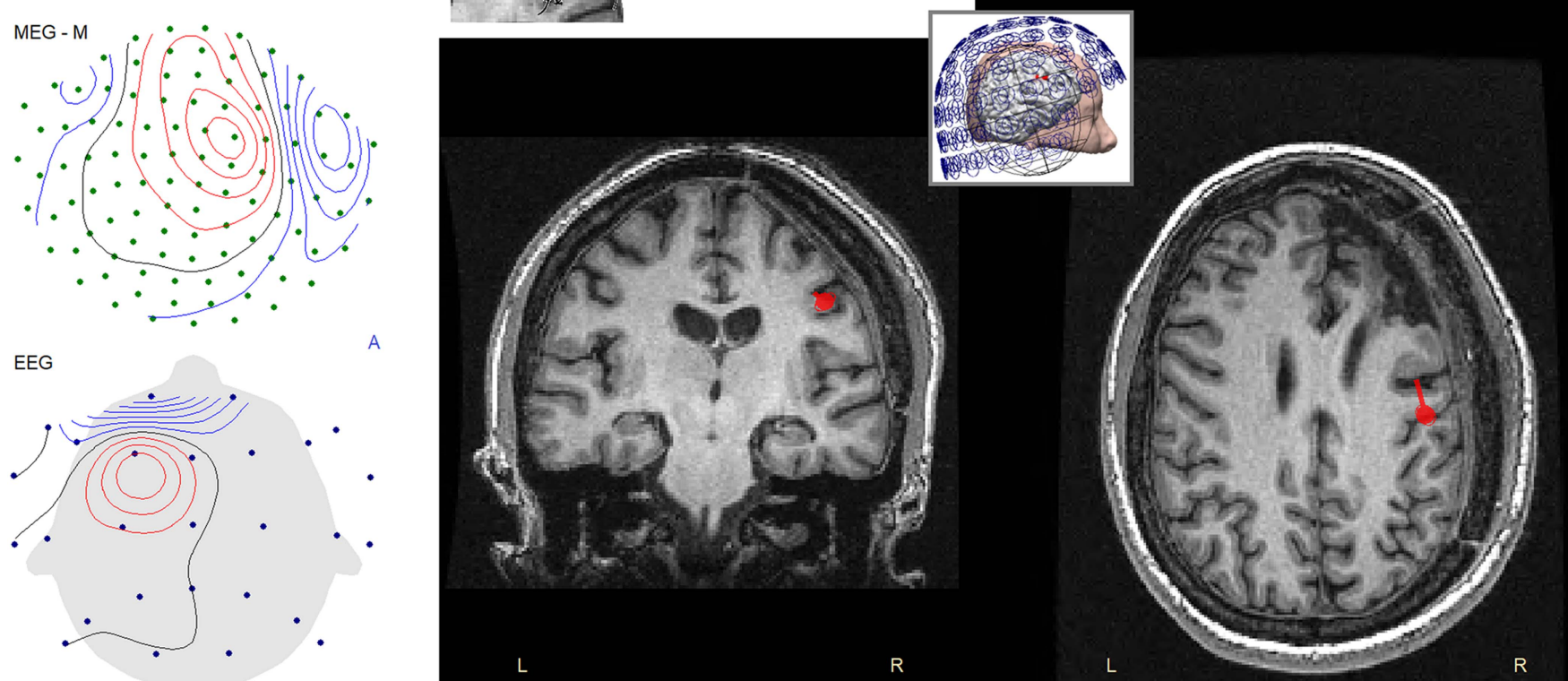

$R / P$

Figure 3: (A) Butterfly plots of back-averaged $(n=790) M E G$ and EEG waveforms (above), averaged on the peak of the myographic potentials at electrode SO1 (upper pink line in EEG trace) and the associated topographic magnetometer flux field and EEG voltage plots at $t=0$ mseconds (below). By the "right-hand rule" of electromagnetism the recorded flux field (red=magnetic flux out of the head, blue= magnetic flux into the head) is attributable to anteriorly directed current flow in the right frontocentral region. The EEG voltage topographic map is dominated by the left frontal electromyographic field (red=electropositive, blue =electronegative). (B) MEG dipole source solution for the back-averaged ( $n=790)$ left orbicularis oculi contractions modeled from -40 mseconds to +4 mseconds, shown on the patient's MRI scan and on a three-dimensional reconstruction of the same MRI scan (the latter viewed directly from the right side (above) and also with the brain slightly rotated (below) to better expose the central sulcus). Red ball=dipole source location; red tail=direction of current flow (away from ball). Explained variance (goodness of $f(t)=87.7 \%$; signal to noise ratio $=10.0$. The source solution is located in the region classically depicted as the eye area of the motor homunculus (compare with the superimposed image of Penfield and Rasmussen's homunculus on the patient's brain MRI scan). Inset shows the patient's head location within the MEG sensor array during the recording as well as the spherical volume conductor. EEG= electroencephalography; G1=planar gradiometers; $\quad G 2=$ planar gradiometers (orthogonal to G1); HFF=high-frequency filter; LFF=low-frequency filter; $M=$ magnetometers $; M E G=$ magnetoencephalography; $M G F P=$ mean global field power. Source modeling of the averaged waveform obtained after exclusion of the 173 potentials recorded during VNS on periods returned a nearly identical solution (Supplementary Fig. S1). 
Magnetoencephalography (MEG) was performed in the hope of identifying a cortical source for the eye twitches and to enable standard MEG source imaging (MSI) of interictal spikes. The 45minute MEG recording was acquired using an Elekta Neuromag TRIUX 306-channel system (Elekta, Helsinki, Finland), with simultaneous 32-channel EEG that included bilateral supraorbital electrodes (SO1/SO2, placed directly inferior to Fp1/Fp2, immediately above the eyebrow). Sampling frequency was $1000 \mathrm{~Hz}$.

To suppress magnetic artifact associated with the VNS, the spatiotemporal signal space separation (tSSS) algorithm ${ }^{1,2}$ implemented within the Elekta Maxfilter system (10-second time window, subspace correlation 0.980) was applied to the data once obtained. As previously reported for active VNS and deep brain stimulator devices, ${ }^{3-5}$ tSSS successfully removed the neurostimulator artifact and permitted easy visual interpretation of the MEG signal, during both on and off phases of the VNS duty cycle (Figure 2).

For MSI analysis of the eye twitching, the MEG signal was first back-averaged on the left orbicularis contractions recorded at SO1 (and Fp1 and F3). The peaks of the myographic potentials were visually identified and manually marked for averaging using CURRY 6 (Compumedics, Abbotsford, Australia). Epochs were generated using a 1 -second time window from -750 to +250 mseconds relative to the myographic peak. Potentials occurring less than 1 second after a marked peak were excluded to permit uncontaminated epoching (see Figure 2, potentials marked by yellow lines selected for averaging). In total, 790 potentials were selected for averaging, 617 (78\%) from VNS off periods and 173 (22\%) from VNS on periods (approximately reflecting the actual soft start VNS duty cycle evident as $25 \mathrm{~Hz}$ artifact in the electrocardiogram (EKG) lead: 172 seconds [81\%] off, 40 seconds [19\%] on).

Interictal spikes were visually identified in the raw EEG and tSSS artifact-suppressed MEG data (band-passed between 1 and $70 \mathrm{~Hz}$ ) and grouped into separate foci for spike averaging based on analysis of each spike's morphology and associated EEG/ MEG voltage/flux field topography, as described previously. ${ }^{5-7} \mathrm{~A}$ total of 55 spikes were identified from a right central focus (C4 EEG maximum; Figure 2) and 22 spikes from a right temporal focus (T4 EEG maximum; Figure 2).

Source modeling of the averaged, epoched data was performed using CURRY 6 as described previously., ${ }^{5,6}$ Noise level was estimated as the variance of the data in the signal from -750 to -250 mseconds before the myographic or interictal spike peaks. A band-pass of 1-30 Hz was used for the eye twitch data and the right central spike focus; a band-pass of 3-30 Hz was used for the right temporal spike focus, where fewer spikes were available for averaging (decreasing signal to noise ratio), and MEG and EEG peaks were asynchronous. A spherical forward model was used for both the eye twitch and interictal spike data. For the eye twitch data, an equivalent current (fixed coherent) dipole inverse model
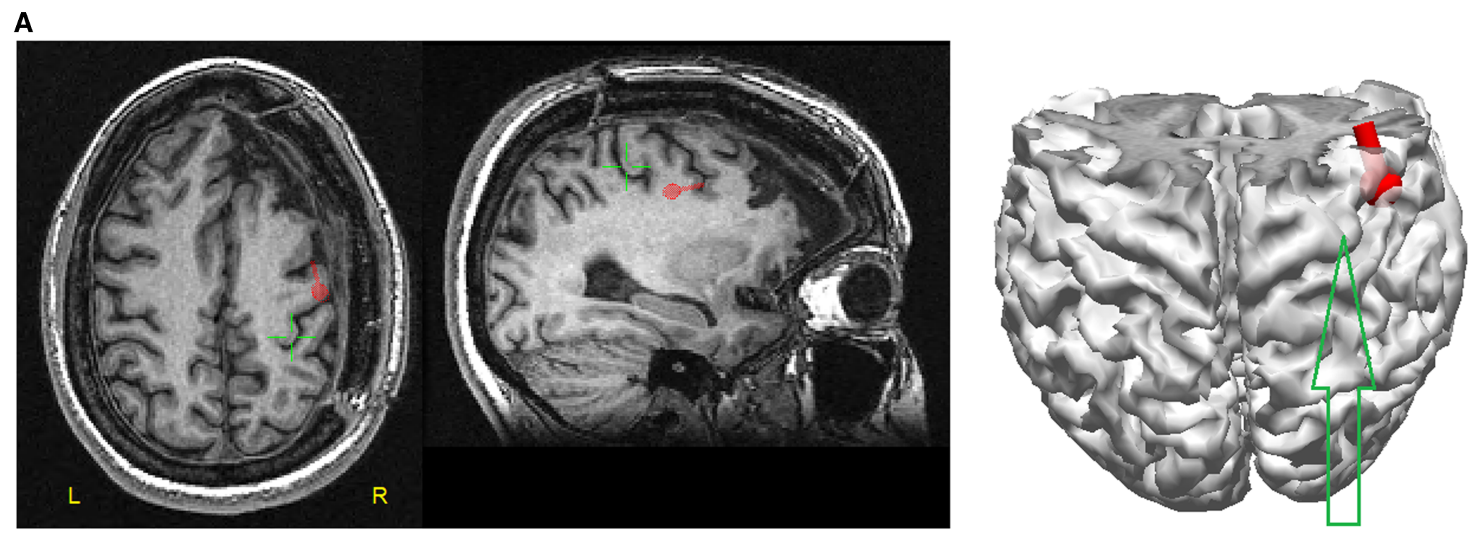

B
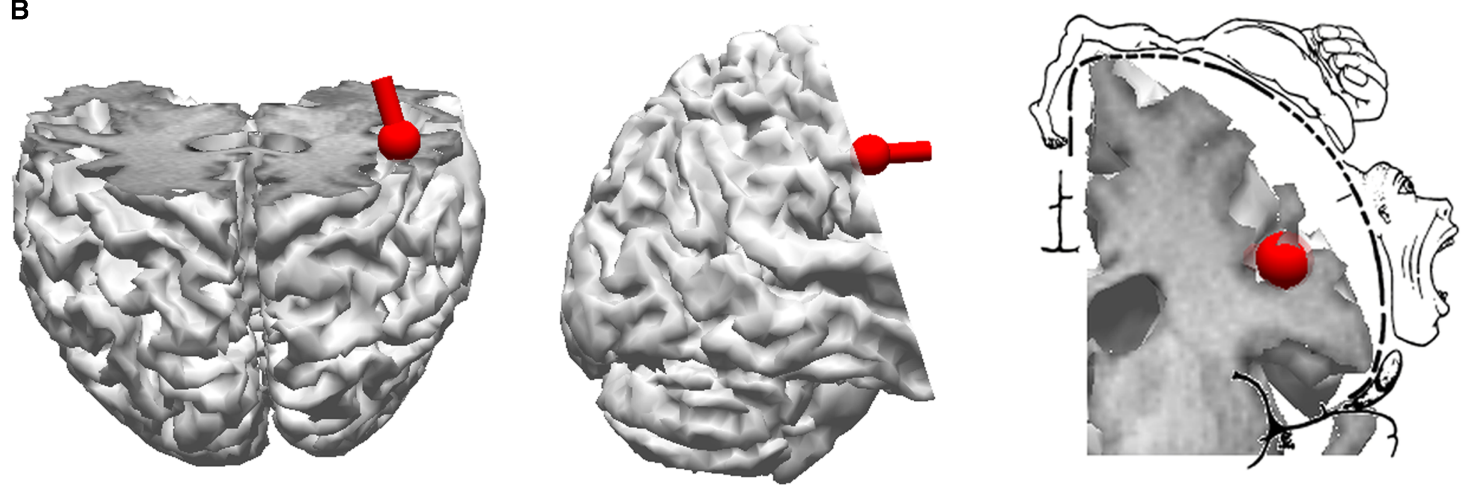

Figure 4: (A) Spatial relation of eye dipole source to the anatomical location of the motor hand area (green cursor and green arrow). The eye dipole source is located lateral and anterior to the anatomical hand area on the posterior aspect of the precentral gyrus. (B) Oblique coronal cut plane through the precentral gyrus at the level of the motor hand area, with superimposition of Penfield and Rasmussen's depiction of the motor homunculus ${ }^{8}$ on this plane of the patient's reconstructed brain MRI scan. 
A

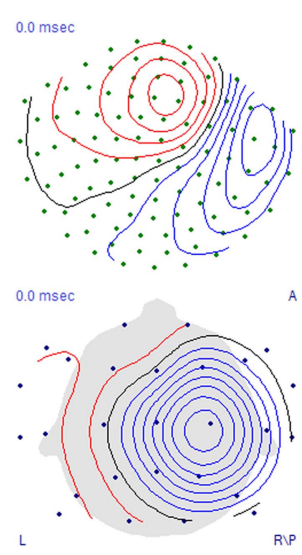

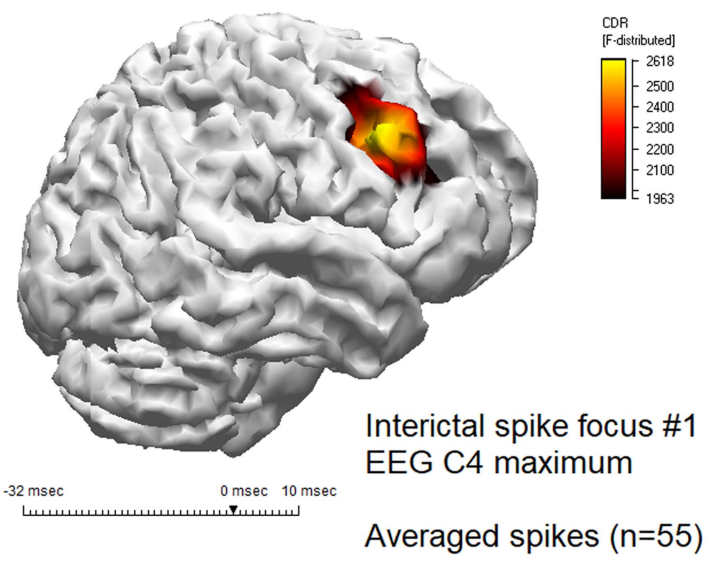

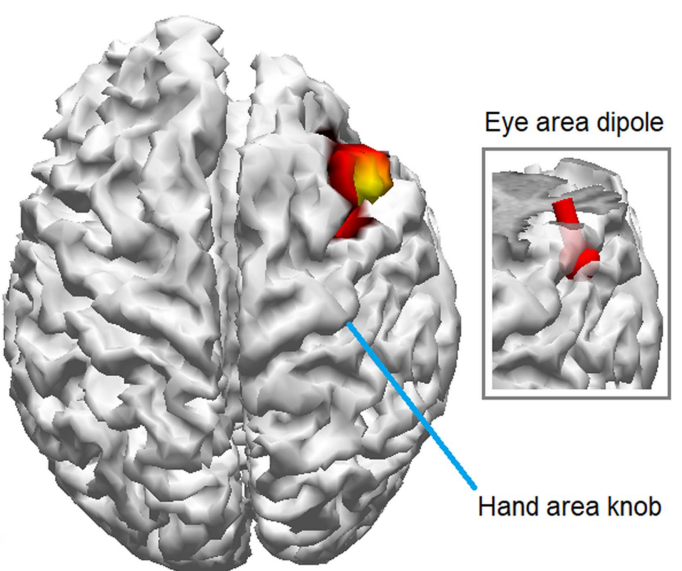

B
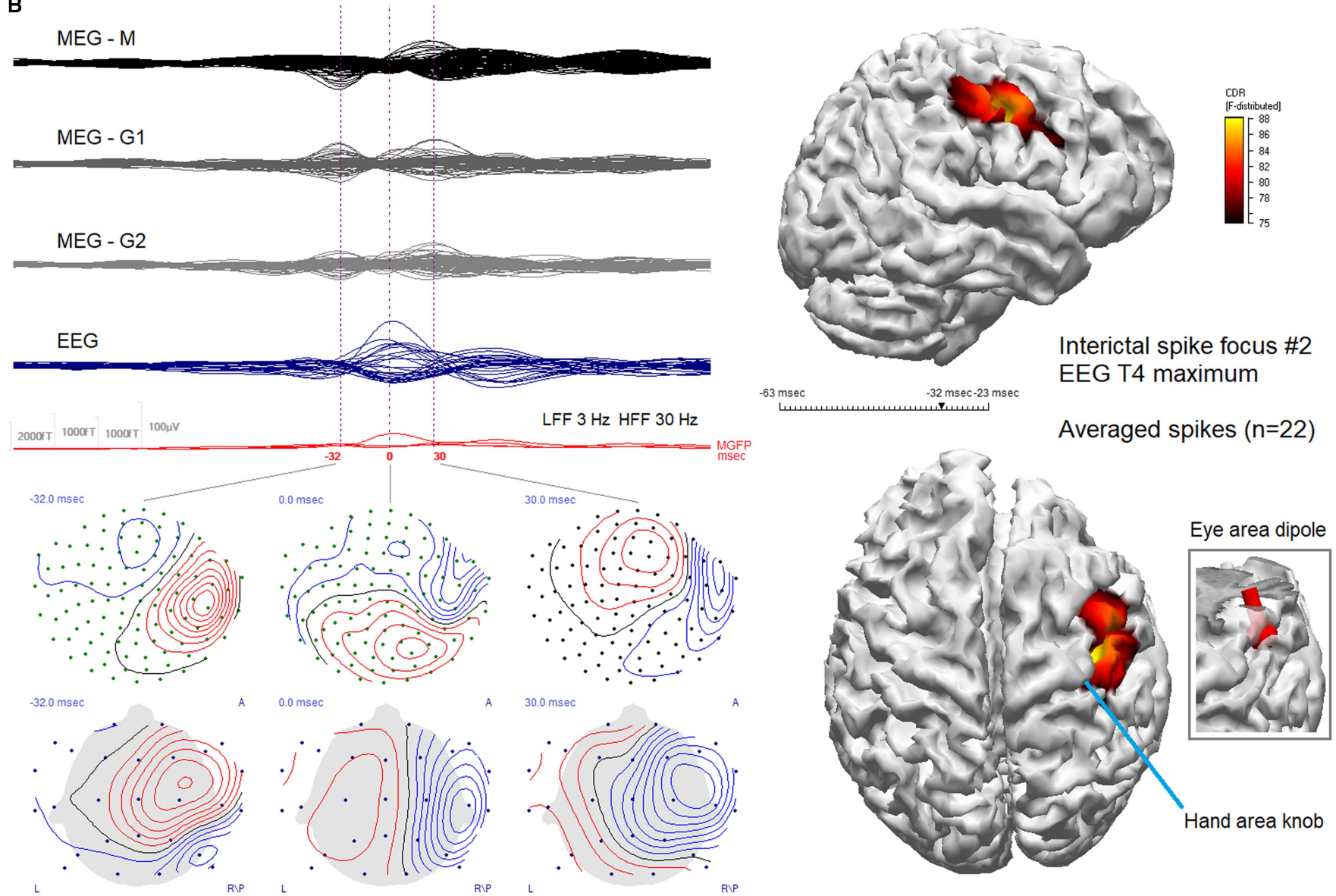

Figure 5: (A) The patient's most active interictal spike focus, with magnetometer flux field topography indicating anteriorly directed current flow associated with the right central EEG voltage topographic maximum at C4 (left). The spike field showed no evidence of propagation over the time period modeled ( -32 mseconds to +10 mseconds; EEG and MEG peaks both at $t=0$ mseconds). sLORETA distributed source solution (middle, right), averaged $(n=55)$ spikes, cortical subspace constraint, rotating sources, $20 \mathrm{~mm}$ extension, solution clipped (threshold) below $75 \%$; signal to noise ratio $=14.0$, explained variance $=99.7 \%$. The source maximum is located one gyrus anterior to the eye area dipole source, in the posterior wall of the gyrus at the edge of the surgical resection. (B) The patient's next most active interictal spike focus, with a propagated multipeak morphology, the first peak apparent in MEG at -32 mseconds, with a magnetometer flux field indicating posteriorly directed current flow, the magnetic field orientation evolving to a transverse direction by $t=0$ mseconds (the T4 EEG voltage peak) and then to an anteriorly directed orientation by +30 mseconds (left). sLORETA distributed source solution for the initial MEG peak (right), modeled from onset to just after peak $(-63$ mseconds to -23 mseconds), averaged $(n=22)$ spikes, cortical subspace constraint, rotating sources, 20 mm extension, solution clipped below 85\%; signal to noise ratio $=4.3$, explained variance $=93.4 \%$. The source maximum of the initial MEG peak is located medial and posterior to the eye area dipole source, within the central sulcus, along the anterior wall of the postcentral gyrus. CDR $=$ current density reconstruction; $E E G=$ electroencephalography; $G 1=$ planar gradiometers; $G 2=$ planar gradiometers (orthogonal to G1); HFF=high-frequency filter; $L F F=$ low-frequency filter; $M=$ magnetometers; $M E G=$ magnetoencephalography; $M G F P=$ mean global field power; sLORETA = standardized low-resolution brain electromagnetic tomography. 
was used. For the interictal spike foci data, a distributed source inverse model was used (standardized low-resolution brain electromagnetic tomography, sLORETA) to highlight the spatial relations between the different source maxima.

The MSI result obtained for the eye twitch data revealed a focal cortical source situated in the posterior wall of the precentral gyrus, in the region of the classical depiction of the eye area of the motor homonculus ${ }^{8}$ (Figure 3 ). The dipole source localization confirmed a clinical suspicion that the patient's eye twitching represented epilepsia partialis continua (EPC) of the orbicularis oculi. The tangential orientation of the dipole source with respect to the cranial surface, with current flowing from posterior to anterior, from superficial to deep within the cortical mantle of the intrasulcal posterior wall of the precentral gyrus (Figure 3), was ideal for detection by MEG, which is most sensitive to tangentially oriented currents. ${ }^{6,7}$ The small size of the source and its intrasulcal location presumably explain in part its invisibility to EEG detection, even after back-averaging and exclusion of the frontal electrodes (data not shown). It is also possible that volume conducted currents from the high-amplitude myographic potentials could eclipse the focal cortical source in EEG, but not MEG, which is insensitive to secondary currents.

Figure 4 shows the eye area dipole source localization in relation to the hand area of the motor homunculus, the latter readily identifiable in MRI scans by a characteristic "knob" in the axial plane and a corresponding "hook" in the sagittal plane. 9 The eye area dipole can be seen to be located lateral and anterior to the hand area, as expected from classical electrical stimulation studies $^{8}$ and from transcranial magnetic stimulation (TMS) studies, where contralateral orbicularis activation occurred in response to TMS applied approximately $2 \mathrm{~cm}$ lateral and $1 \mathrm{~cm}$ anterior to the motor hand area. ${ }^{10}$

With regard to the incomplete right orbicularis contractions seen in association with the left eye twitches (with eyes open; Figure 1, and video), the most parsimonious explanation is that the right-sided twitches reflect ipsilateral projections from the right motor cortex (in keeping with the traditional neurological concept of bilateral cortical innervation of the upper facial muscles).

With regard to the patient's persistent major motor seizures (which are independent of her new onset EPC), ictal onsets during the EMU admission were poorly and inconsistently localized, usually showing a right centroparietal maximum. The MSI of her most active interictal spike foci revealed the C4 EEG spikes to be located anterior to the eye area dipole source, abutting the resection margin, whereas the T4 EEG spikes were found to have a complicated pattern of propagation with an initial MEG peak 30 mseconds before the EEG peak localized within the central sulcus, just medial and posterior to the eye area dipole source along the anterior wall of the postcentral gyrus (Figure 5). Future medical or surgical treatment possibilities are under discussion with the patient.

The classical depiction of the motor homunculus defined by intraoperative electrical stimulation of the precentral gyrus includes an eye area. ${ }^{8}$ Notwithstanding, TMS investigations have questioned whether projections exist from primary motor cortex to the upper facial muscles in man, although one study clearly showed contralateral orbicularis activation in response to TMS applied anterolateral to the motor hand area. ${ }^{10}$ The discrete localization by MSI of the cortical generator responsible for this patient's orbicularis EPC provides novel, further support for the existence of a human motor homuncular eye area.

\section{ACKNOWLEDGMENTS}

The authors express their gratitude to Nat Shampur for expert technical assistance and to the patient for her willingness to permit the presentation of her clinical investigations.

\section{Statement of Authorship}

JMdC and RW conceived the study. LGD and RW acquired and analyzed the MEG data. RW prepared the figures and wrote the manuscript. All authors critically reviewed the manuscript and approved the final version.

\section{DISCLOSURE INFORMATION}

Richard Wennberg, Luis Garcia Dominguez, and J. Martin del Campo do not have any disclosures.

\section{SuPPlementary Material}

To view supplementary material for this article, please visit https://doi.org/10.1017/cjn.2018.373

\section{REFERENCES}

1. Taulu S, Simola J. Spatiotemporal signal space separation method for rejecting nearby interference in MEG measurements. Phys Med Biol. 2006;51:1759-68.

2. Taulu S, Hari R. Removal of magnetoencephalographic artifacts with temporal signal-space separation: demonstration with singletrial auditory-evoked responses. Hum Brain Mapp. 2009;30:1524-34.

3. Tanaka N, Thiele EA, Madsen JR, et al. Magnetoencephalographic analysis in patients with vagus nerve stimulator. Pediatr Neurol. 2009;41:383-7.

4. Carrette E, De Tiège X, De Beeck MO, et al. Magnetoencephalography in epilepsy patients carrying a vagus nerve stimulator. Epilepsy Res. 2011;93:44-52.

5. Wennberg R, del Campo JM, Shampur N, et al. Feasibility of magnetoencephalographic source imaging in patients with thalamic deep brain stimulation for epilepsy. Epilepsia Open. 2016;2:101-6.

6. Wennberg R, Cheyne D. Reliability of MEG source imaging of anterior temporal spikes: analysis of an intracranially characterized spike focus. Clin Neurophysiol. 2014;125:211-5.

7. Wennberg R, Cheyne D. Elucidating the meaning of dipole variability in MEG/MSI. Clin Neurophysiol. 2015;126:211-5.

8. Penfield W, Rasmussen TL. The cerebral cortex of man: A clinical study of localization of function. New York: Macmillan; 1950.

9. Yousry TA, Schmid UD, Alkadhi H, et al. Localization of the motor hand area to a knob on the precentral gyrus: A new landmark. Brain. 1997;120:141-57.

10. Paradiso GO, Cunic DI, Gunraj CA, Chen R. Representation of facial muscles in human motor cortex. J Physiol. 2005;567: 323-36. 